\title{
Adaptive Pinning Synchronization Control of the Fractional-Order Chaos Nodes in Complex Networks
}

\author{
Darui Zhu, ${ }^{1,2}$ Ling Liu, ${ }^{1,2}$ and Chongxin Liu ${ }^{1,2}$ \\ ${ }^{1}$ State Key Laboratory of Electrical Insulation and Power Equipment, Xian Jiaotong University, Xian 710049, China \\ ${ }^{2}$ School of Electrical Engineering, Xian Jiaotong University, Xi'an 710049, China \\ Correspondence should be addressed to Ling Liu; liul@mail.xjtu.edu.cn
}

Received 4 November 2013; Revised 15 March 2014; Accepted 16 April 2014; Published 5 May 2014

Academic Editor: Kwok-Wo Wong

Copyright (c) 2014 Darui Zhu et al. This is an open access article distributed under the Creative Commons Attribution License, which permits unrestricted use, distribution, and reproduction in any medium, provided the original work is properly cited.

\begin{abstract}
Adaptive pinning synchronization control is studied for a class of fractional-order complex network systems which are constructed depending on small-world network algorithm. Based on the fractional-order stability theory, the suitable adaptive control scheme is designed to guarantee global asymptotic stability of all the nodes in complex network systems and the node selected algorithm is given. In numerical implementation, it is shown that the numerical solution of the fractional-order complex network systems can be obtained by applying an improved version of Adams-Bashforth-Moulton algorithm. Furthermore, simulation results are provided to confirm the validity and synchronization performance of the advocated design methodology.
\end{abstract}

\section{Introduction}

Fractional-order calculus can portray the physical phenomenon more accurately than integer order calculus in actual system. In recent years, the theoretical analysis and applied research of the fractional calculus have attracted many researchers' attentions. Fractional-order calculus has become a hot research topic in dynamic system, and there are many successful applications in engineering field [15]. Some scholars found that the fractional-order nonlinear dynamic system also shows chaotic phenomenon, such as fractional-order Rössler system, fractional-order Liu system, and fractional-order Chen system [6-13].

In nature, many complex systems can be described as complex networks. Complex networks consist of nodes and the connecting relations of nodes component. Over the past twenty years, complex networks have been widely studied in various fields such as large-scale circuit networks, power networks, computer networks, communication networks, and automatic control systems [14-16]. Complex networks have become an important research topic in these years. And many theoretical analyses of the characteristics are studied in the network [17-21].
Many synchronization control methods of the integer order nodes in complex networks are studied, such as cluster synchronization, adaptive control synchronization, pinning control synchronization, projective synchronization, robust impulsive synchronization [22-26]. Because of the superiority of the fractional calculus in the description of physical phenomena, considering fractional-order systems as nodes in complex networks has important theoretical significance. In recent years, some synchronization control methods of fractional-order chaotic systems in complex networks are studied. The adaptive control methods are used in complex networks [27], the cluster synchronization in complex networks [28], the robust outer synchronization between two complex networks [29], and the generalized synchronization of the fractional-order chaos in weighted complex dynamical networks with nonidentical nodes [30]. In this paper, we proposed a novel synchronization theorem for fractionalorder complex networks. Based on the proposed control scheme, the adaptive feedback controllers are designed for pinning control. Theoretical analyses show that the suitable adaptive feedback gains achieved all the nodes synchronized with each other in the complex networks. 
The rest of this paper is organized as follows. In Section 2, some important preliminaries are introduced for demonstrating the main results in the following sections. In Section 3, an effective adaptive pinning control scheme of the fractionalorder complex networks is studied and the theoretical proof is given. In Section 4, the control scheme is applied to the fractional-order chaotic systems in complex networks, and the simulation results are given to demonstrate the theoretical analysis. Conclusions ended the paper in Section 5.

\section{Model Description and Preliminaries}

At present, there are several definitions of the fractional-order differential systems. Two commonly used definitions are Grünwald-Letnikov (GL) definition and Riemann-Liouville (RL) definition.

The best-known RL definition of fractional-order can be expressed as [1]

$$
\frac{\mathrm{d}^{q} f(t)}{\mathrm{d} t^{q}}=\frac{1}{\Gamma(n-q)} \frac{\mathrm{d}^{n}}{\mathrm{~d} t^{n}} \int_{0}^{t} \frac{f(\tau)}{(t-\tau)^{q-n+1}} d \tau,
$$

where $n$ is an integer satisfying $n-1 \leq q<n$ and $\Gamma(\cdot)$ is the $\Gamma$-function.

Considering a general fractional-order nonlinear dynamical system as follows:

$$
D^{q} X=f(X) \quad \text { or } \quad D^{q} X=A X,
$$

where $X \in R^{n}(\in N), A \in R^{n \times n}, 0<q \leq 1$.

Lemma 1. For a given autonomous linear system of fractionalorder system (2) with $x(0)=x_{0}$, where $x(t) \in R^{n}$ is the state vector, from paper [31], we know the following.

(1) The system is asymptotically stable if and only if $\left|\arg \left(\lambda_{i}(A)\right)\right|>\alpha \pi / 2, i=1,2, \ldots, n$, where $\arg \left(\lambda_{i}(A)\right)$ denotes the argument of the eigenvalues $\lambda_{i}$ of $A$.

(2) The system is stable if and only if either it is asymptotically stable or those critical eigenvalues which satisfying $\left|\arg \left(\lambda_{i}(A)\right)\right|=\alpha \pi / 2$ have geometric multiplicity one.

Lemma 2 (see [32, 33]). For the nonlinear fractional-order system (2) with the order as $0<q \leq 1$, if there exists a real symmetric positive definite matrix $P$ satisfying $J(t)=$ $X^{T}(t) P D^{q} X(t) \leq 0$, where $X(t)=\left(x_{1}(t), x_{2}(t), \ldots, x_{n}(t)\right)$, then system (2) is asymptotically locally stable.

Consider that the generic dynamical complex networks model consists of $N$ coupled identical nodes, and each node is an n-dimensional dynamical system. The fractional-order network model is described as follows [34, 35]:

$$
\frac{\mathrm{d}^{q} x_{i}(t)}{\mathrm{d} t^{q}}=f\left(x_{i}(t)\right)+c \sum_{j=1}^{N} g_{i j} \Gamma x_{j}(t), \quad i=1,2, \ldots, N
$$

where $x_{i}(t)=\left(x_{i 1}(t), x_{i 2}(t), \ldots, x_{i N}(t)\right)^{T} \in R^{n}$ represent the $i$ th node of the $n$-dimensional dynamical system. $\Gamma$ is the innercoupling term which links the coupled variables in networks.
The constant $c>0$ is the coupling strength. $G=\left(g_{i j}\right)_{N \times N}$ is the coupling configuration matrix of any of the two nodes, in which $g_{i j}$ is defined as follows. If there is a connection between the ith node and the $j$ th node, $(j \neq i)$, then $g_{i j}=g_{j i}=1$; otherwise, $g_{i j}=g_{j i}=0(j \neq i)$, and the diagonal elements of matrix $G$ are defined by

$$
g_{i i}=-\sum_{\substack{j=1 \\ j \neq i}}^{N} g_{i j}=-\sum_{\substack{j=1 \\ j \neq i}}^{N} g_{j i}, \quad i=1,2, \ldots, N
$$

Definition 3. If $x_{1}(t)=x_{2}(t)=\cdots=x_{N}(t) \rightarrow s(t)$, $t \rightarrow \infty$. Consider $\lim _{t \rightarrow \infty}\left\|x_{i}(t)-s(t)\right\|=0(1 \leq i \leq N)$, the complex network is achieved synchronization.

Where $s(t) \in R^{n}$ is a solution of an isolated node, satisfying

$$
D^{q} s(t)=f[s(t)],
$$

$s(t)$ can be an equilibrium point, a periodic orbit, or even a chaotic attractor.

The error vector is defined as

$$
e_{i}(t)=x_{i}(t)-s(t), \quad(i=1,2, \ldots, N) .
$$

Assumption 4 (see [36]). Suppose that there exists a nonnegative constant $\alpha, \Gamma$ is a symmetric positive semidefinite matrix, and arbitrary $x, y \in R^{n}$, such that

$$
\begin{array}{r}
(x-y)^{T}(f(x)-f(y)) \leq(x-y)^{T} \alpha \Gamma(x-y) . \\
\forall x, y \in R^{n} .
\end{array}
$$

Lemma 5 (see [37]). The linear matrix inequality (LMI) $S=$ $\left[\begin{array}{ll}S_{11} & S_{12} \\ S_{12}^{T} & S_{22}\end{array}\right]<0$ is equivalent to any one of the two following conditions:

$$
\text { (1) } S_{11}<0, S_{22}-S_{12}^{T} S_{11}^{-1} S_{12}<0 \text {, }
$$$$
\text { (2) } S_{22}<0, S_{11}-S_{12} S_{22}^{-1} S_{12}^{T}<0 \text {, }
$$

where $S_{11}=S_{11}^{T}$ and $S_{22}=S_{22}^{T}$.

\section{Adaptive Pinning Synchronization}

In this section, a novel adaptive pinning control scheme is proposed for the synchronization in complex networks, and this method is proved based on the fractional-order stability theory. The controlled fractional-order complex network model can be described in the following equations:

$$
\begin{aligned}
& \frac{\mathrm{d}^{q} x_{i}(t)}{\mathrm{d} t^{q}} \\
& \quad\left\{\begin{array}{l}
f\left(x_{i}(t)\right) \\
+c \sum_{j=1}^{N} g_{i j} \Gamma x_{j}(t)+u_{i}(t), \quad i=1,2, \ldots, l, \\
f\left(x_{i}(t)\right)+c \sum_{j=1}^{N} g_{i j} \Gamma x_{j}(t), \quad i=l+1, l+2, \ldots, N .
\end{array}\right.
\end{aligned}
$$


The adaptive pinning controllers and the updating laws are defined as

$$
\begin{gathered}
u_{i}(t)=-d_{i}(t) \Gamma e_{i}(t), \quad i=1,2, \ldots, l, \\
\frac{\mathrm{d}^{q} d_{i}(t)}{\mathrm{d} t^{q}}=k_{i} e_{i}^{T}(t) \Gamma e_{i}(t), \quad i=1,2, \ldots, l, \\
u_{i}(t)=0, \quad i=l+1, l+2, \ldots, N,
\end{gathered}
$$

where $k_{i}>0$ is an arbitrary constant, the complex network realized pinning synchronization based on the above adaptive controllers.

Based on the above equations, we can get the error equations as follows:

$$
\begin{aligned}
& \frac{\mathrm{d}^{q} e_{i}(t)}{\mathrm{d} t^{q}} \\
& = \begin{cases}f\left(x_{i}(t)\right)-f(s(t)) & \\
+c \sum_{j=1}^{N} g_{i j} \Gamma e_{j}(t)-d_{i}(t) \Gamma e_{i}(t) & i=1,2, \ldots, l, \\
f\left(x_{i}(t)\right)-f(s(t)) & \\
+c \sum_{j=1}^{N} g_{i j} \Gamma e_{j}(t), & i=l+1, l+2, \ldots, N .\end{cases}
\end{aligned}
$$

Theorem 6. The complex networks (8) are realized adaptive pinning global synchronizations under the controllers (9), which is satisfied in $\lambda(G)_{N-l}<-\alpha / c$, and $d^{*}>\lambda_{\max }\left(S_{11}-\right.$ $\left.S_{12} S_{N-l}^{-1} S_{12}^{T}\right)$, where $\widetilde{I}_{N}=\operatorname{diag}(\overbrace{1, \ldots, 1}^{l}, \overbrace{0, \ldots, 0}^{N-l})$ and $d^{*}=$ $\min _{1 \leq i \leq l}\left\{d_{i}^{*}\right\}$.

Proof. The error system (10) can realize asymptotic stability at the state $x(t)=s(t)$, denote $\widetilde{d}_{i}(t)=d_{i}(t)-d^{*}$, and $d^{*}$ is a positive constant which need to be determined.

Further denote $X(t)=(E(t), \widetilde{d}(t))^{T}$, where

$$
\begin{gathered}
E(t)=\left(e_{1}(t), e_{2}(t), \ldots, e_{N}(t)\right)^{T}, \\
e_{i}=\left(e_{i 1}(t), e_{i 2}(t), \ldots, e_{i N}(t)\right)^{T}, \\
\widetilde{d}(t)=\left(\tilde{d}_{1}(t), \tilde{d}_{2}(t), \ldots, \tilde{d}_{N}(t)\right)^{T} .
\end{gathered}
$$

Choose the real symmetric positive definite matrix $P$ as

$$
P=\operatorname{diag}(\overbrace{1, \ldots, 1}^{N}, \frac{1}{k_{1}}, \frac{1}{k_{2}}, \ldots, \frac{1}{k_{N}}) .
$$

We obtain that

$$
\begin{aligned}
J(t)= & X^{T}(t) P D^{q} X(t)=\sum_{i=1}^{N} e_{i}^{T}(t) D^{q} e_{i}(t) \\
& +\sum_{i=1}^{N} \widetilde{d}_{i} \frac{1}{k_{i}} D^{q} \widetilde{d}_{i}(t) \\
= & \sum_{i=1}^{N} e_{i}^{T}(t) D^{q} e_{i}(t)+\sum_{i=1}^{N} \frac{1}{k_{i}}\left(d_{i}-d_{i}^{*}\right) k_{i} e_{i}^{T}(t) \Gamma e_{i}(t)
\end{aligned}
$$

$$
\begin{aligned}
& =\sum_{i=1}^{N} e_{i}^{T}(t)\left[\left(f\left(x_{i}(t)\right)-f(s(t))\right)\right. \\
& \left.+c \sum_{j=1}^{N} g_{i j} \Gamma\left(x_{j}(t)-s(t)\right)-d_{i} \Gamma e_{i}(t)\right] \\
& +\sum_{i=1}^{l}\left(d_{i}-d_{i}^{*}\right) e_{i}^{T}(t) \Gamma e_{i}(t) \\
& \leq \alpha \sum_{i=1}^{N} e_{i}^{T}(t) \Gamma e_{i}(t)+c \sum_{i=1}^{N} \sum_{j=1}^{N} e_{i}^{T}(t) g_{i j} \Gamma e_{j}(t) \\
& -d_{i}^{*} \sum_{i=1}^{l} e_{i}^{T}(t) \Gamma e_{i}(t) \\
& =e^{T}(t)\left[\left(\alpha I_{N}-D_{N}+c G\right) \otimes \Gamma\right] e(t) \\
& =e^{T}(t)(Q \otimes \Gamma) e(t),
\end{aligned}
$$

where $\otimes$ is Kronecker product, $Q=\alpha I_{N}+c G-D_{N}$, and $D_{N}=\operatorname{diag}(\overbrace{1, \ldots, 1}^{l}, \overbrace{0, \ldots, 0}^{N-l})$. Let $S=\alpha I_{N}+c G$; that is, $Q=S-D_{N}$. By Lemma $5, S-D_{N}=\left[\begin{array}{cc}S_{11}-D_{l} & S_{12} \\ S_{12}^{T} & S_{N-l}\end{array}\right]$, where $D_{l}=\operatorname{diag}\left(d_{1}^{*}, \ldots, d_{l}^{*}\right)$. Consider $\lambda\left(S_{N-l}\right)=\lambda\left(\alpha I_{N}+c G\right)_{N-l} \leq$ $\alpha+c \lambda(G)_{N-l}$, and from Theorem $6, \alpha+c \lambda(G)_{N-l}<0$, $\lambda(G)_{N-l}<-\alpha / c$, we can get $S_{N-l}<0$. By Lemma 5 , if $S<0$, that is, $\left(S_{11}-D_{l}\right)-S_{12} S_{N-l}^{-1} S_{12}^{T}<0, D_{l}>S_{11}-S_{12} S_{N-l}^{-1} S_{12}^{T}$, which indicates that $d^{*}>\lambda_{\max }\left(S_{11}-S_{12} S_{N-l}^{-1} S_{12}^{T}\right)$, where $d^{*}=$ $\min _{1 \leq i \leq l}\left\{d_{i}^{*}\right\}$. There exists a suitable $\alpha$ to satisfy Assumption 4 and $d^{*}$ is large enough to make the $Q<0$, and $\Gamma$ is a positive semidefinite matrix; it is easy to see that $Q \otimes \Gamma \leq 0$. According to the fractional-order stability theory, the system is asymptotically stable. Then the proof is completed.

Proposition 7. If the matrix $\alpha I_{N}+c G$ has $m$ nonnegative eigenvalues, and if $\alpha I_{N}+c G-D_{N}<0$, then the number of nodes to be selected for control cannot be less than $\mathrm{m}$.

Proof. By the inequality $Q=\alpha I_{N}+c G-D_{N}<0$, we can get $Q_{i i}<0, Q_{i i}=\alpha+c g_{i i}-d_{i}<0$, and the degree of the networks $\operatorname{Deg}_{i}=-g_{i i}$, so $\alpha-c \operatorname{Deg}_{i}-d_{i}<0, \operatorname{Deg}_{i}>\left(\alpha-d_{i}\right) / c(i=$ $1, \ldots, l), \operatorname{Deg}_{i}>\alpha / c(i=l+1, \ldots, N)$.

For the above theoretical proof, we can obtain that if the coupling strength $c$ is large enough, selecting the arbitrary $l$ nodes is satisfied. But when the coupling strength $c$ is small, we need to select the small node degree nodes to add the controllers.

\section{Simulation Results}

To demonstrate the effectiveness of the proposed approach, the fractional-order Liu chaotic system as node in the complex network systems is applied to construct the network model with 50 nodes based on the NW small-world network 
algorithm. The controlled nodes in the complex networks can be written as follows:

$$
\begin{aligned}
\frac{\mathrm{d}^{q} x_{i 1}(t)}{\mathrm{d} t^{q}}= & 10\left(x_{i 2}(t)-x_{i 1}(t)\right) \\
& +c \sum_{j=1}^{N} g_{i j} x_{j 1}(t)-d_{i}(t) \Gamma\left(x_{i 1}(t)-s(t)\right), \\
\frac{\mathrm{d}^{q} x_{i 2}(t)}{\mathrm{d} t^{q}}= & 40 x_{i 1}(t)-x_{i 1}(t) x_{i 3}(t) \\
& +c \sum_{j=1}^{N} g_{i j} x_{j 2}(t)-d_{i}(t) \Gamma\left(x_{i 2}(t)-s(t)\right), \\
\frac{\mathrm{d}^{q} x_{i 3}(t)}{\mathrm{d} t^{q}=} & -2.5 x_{i 3}(t)+4 x_{i 1}(t) x_{i 1}(t) \\
& +c \sum_{j=1}^{N} g_{i j} x_{j 3}(t)-d_{i}(t) \Gamma\left(x_{i 3}(t)-s(t)\right) .
\end{aligned}
$$

In order to achieve the synchronization control of the above system, the Adams-Bashforth-Moulton algorithm is applied to the system (14). Consider the following differential equations:

$$
\begin{gathered}
{ }_{0}^{C} D_{t}^{\alpha} y_{i}(t)=f\left(t, y_{i}(t)\right)+c \sum_{\substack{j=1 \\
j \neq i}}^{N} g_{i j} \Gamma y_{j}(t), \quad 0 \leq t \leq T, \\
y_{i}^{(k)}(0)=y_{i 0}^{(k)}, \quad k=0,1, \ldots,[\alpha]-1, i=1,2, \ldots, N .
\end{gathered}
$$
$[1,38]$

It is equivalent to the Volterra integral equation as follows

$$
\begin{aligned}
& y_{i}(t) \\
& =\sum_{k=0}^{[\alpha]-1} \frac{t^{k}}{k !} y_{i 0}^{(k)}+\frac{1}{\Gamma(\alpha)} \\
& \quad \times\left(\int _ { 0 } ^ { t } ( t - w ) ^ { \alpha - 1 } \left(f\left(w, y_{i}(w)\right)\right.\right. \\
& \left.\left.+c \sum_{j=1}^{N} g_{i j} \Gamma y_{j}(w)\right) d w\right) .
\end{aligned}
$$

Here, $h=T / N, t_{n}=n h, n=0,1, \ldots, N \in Z$, and (16) can be discretized into [9]

$$
\begin{aligned}
y_{i h}\left(t_{n+1}\right)= & \sum_{k=0}^{[\alpha]-1} \frac{t_{n+1}^{k}}{k !} y_{i 0}^{(k)}+\frac{h^{\alpha}}{\Gamma(\alpha+2)} \\
& \times\left(f\left(t_{n+1}, y_{i h}^{*}\left(t_{n+1}\right)+c \sum_{j=1}^{N} g_{i j} \Gamma y_{j h}\left(t_{n+1}\right)\right)\right)
\end{aligned}
$$

$$
\begin{array}{r}
+\frac{h^{\alpha}}{\Gamma(\alpha+2)} \sum_{l=0}^{n} a_{l, n+1}\left(f \left(t_{l}, y_{i h}\left(t_{l}\right)\right.\right. \\
\left.\left.+c \sum_{j=1}^{N} g_{i j} \Gamma y_{j h}\left(t_{l}\right)\right)\right), \\
i=1,2, \ldots, N,
\end{array}
$$

where

$$
\begin{aligned}
a_{l, n+1}= & \begin{cases}n^{\alpha+1}-(n-\alpha)(n+1)^{\alpha} & l=0 \\
(n-l+2)^{\alpha+1}+(n-l)^{\alpha+1} & \\
-2(n-l+1)^{\alpha+1} & 1 \leq l \leq n \\
1 & l=n+1,\end{cases} \\
y_{i h}^{*}\left(t_{n+1}\right)= & \sum_{k=0}^{[\alpha]-1} \frac{t_{n+1}^{k}}{k !} y_{i 0}^{(k)}+\frac{1}{\Gamma(\alpha)} \\
& \times \sum_{l=0}^{n} b_{l, n+1}\left(f\left(t_{l}, y_{i h}\left(t_{l}\right)+c \sum_{j=1}^{N} g_{i j} \Gamma y_{j h}\left(t_{l}\right)\right)\right), \\
& b_{l, n+1}=\frac{h^{\alpha}}{\alpha}\left((n+1-l)^{\alpha}-(n-l)^{\alpha}\right) .
\end{aligned}
$$

The error equation is $\max _{j=0,1, \ldots, N}\left|y\left(t_{j}\right)-y_{h}\left(t_{j}\right)\right|=O\left(h^{p}\right)$, where $p=\min (2,1+\alpha)$.

Where $g_{i j}$ is the coupling configuration matrix which is generated by the NW small-world algorithm, $c$ is the coupling strength, and the inner-coupling term $\Gamma=\left(\begin{array}{llll}1 & 0 & 0 & 0 \\ 0 & 1 & 0 & 0 \\ 0 & 0 & 1 & 0 \\ 0 & 0 & 0 & 1\end{array}\right)$. When the fractional-order $0.83 \leq q \leq 1$, the $i$ th node of the fractional-order Liu chaotic system (14) is chaotic [10]. Figure 1 shows the chaotic attractor of the fractional-order Liu system with the order $q=0.9$.

In the following, we choose $\alpha=135$, and coupling strength $c=12$. Select the initial value $x_{i 1}=20 \times$ rand, $x_{i 2}=20 \times$ rand, and $x_{i 3}=20 \times$ rand, where rand $\epsilon$ $(0,1)$ is a random number, and $d^{*}=2$. When $l=20$, $\lambda(G)_{N-l}=-11.9644$, which is satisfied in $\lambda(G)_{N-l}<-\alpha / c=$ -11.25 . That is, we need to pin 20 nodes for achieving synchronization in the networks.

The synchronization errors are defined as (10), and the total synchronization error is defined as $E(t)=$ $(1 / N) \sqrt{\sum_{i=1}^{N}\left(e_{i 1}^{2}+e_{i 2}^{2}+e_{i 3}^{2}\right)}$. Control-effort trajectory of error evolutions is shown in Figures 2 and 3 . These figures show that the nodes of the networks are globally asymptotically stable under the pinning adaptive controllers. The evolutions of the pinning feedback gains with (9) are illustrated in Figure 4.

\section{Conclusions}

In this paper, a novel adaptive pinning control scheme is proposed to deal with chaos synchronization for a class 


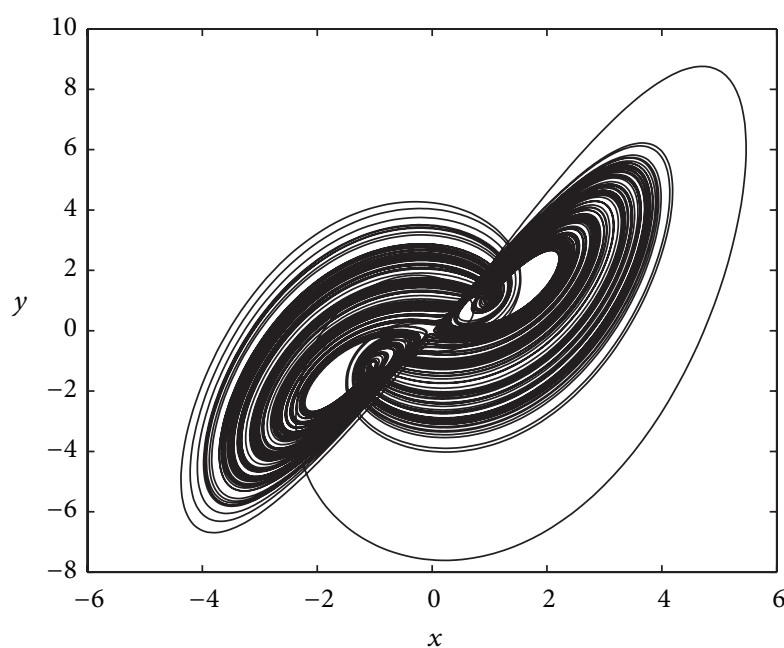

(a) $x-y$ phase diagram

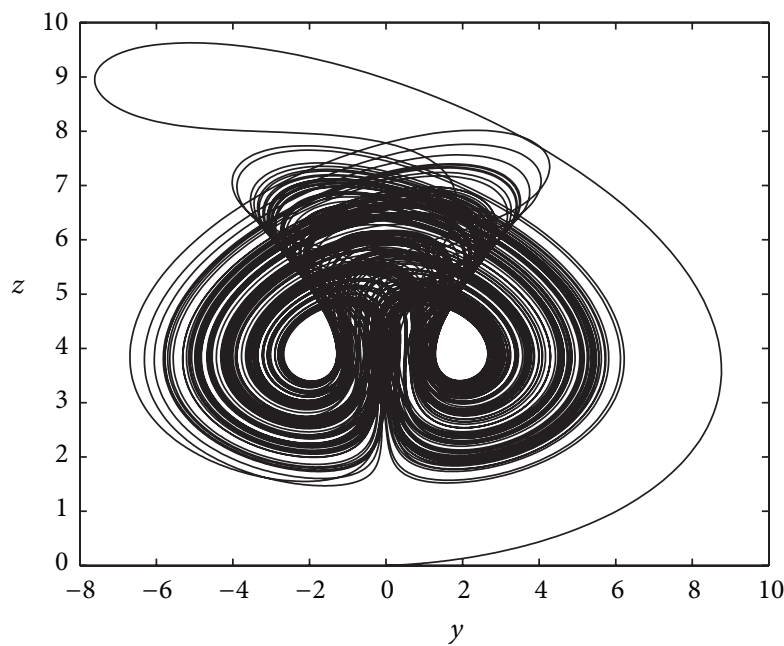

(b) $y-z$ phase diagram

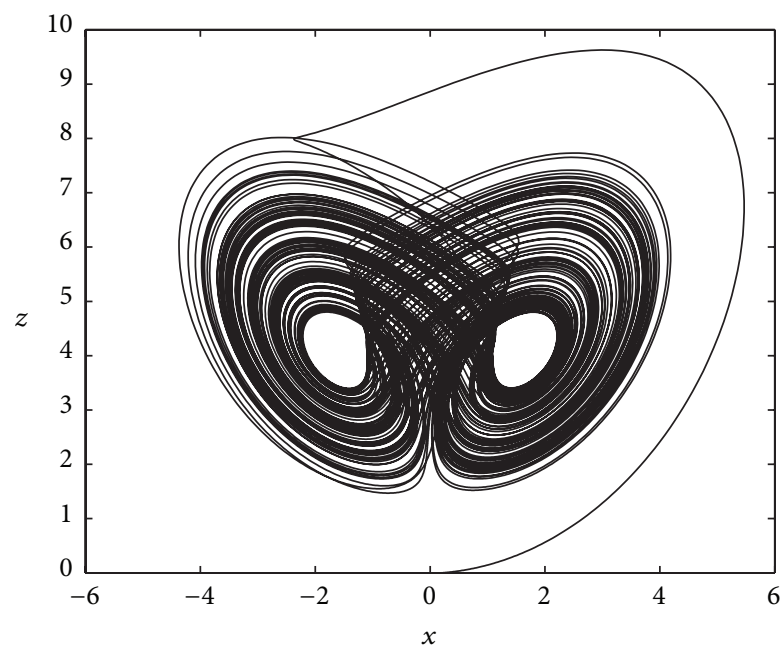

(c) $x-z$ phase diagram

FIGURE 1: 2D strange attractors of the fractional-order Liu chaotic system.
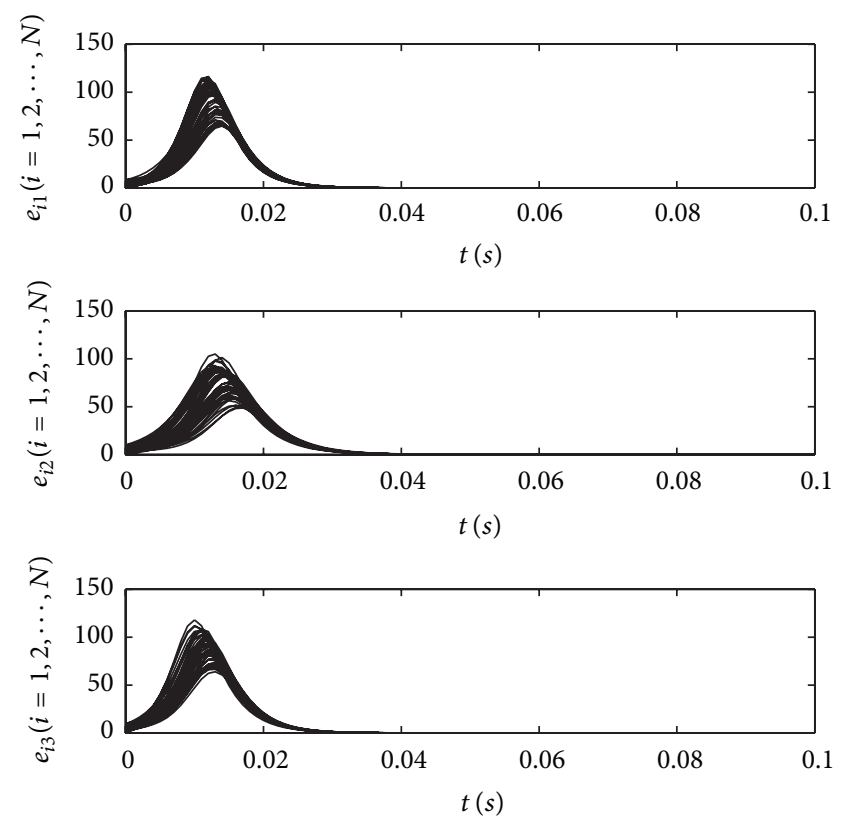

Figure 2: Synchronization errors of $e_{i 1}, e_{i 2}, e_{i 3}, 1 \leq i \leq 50$.

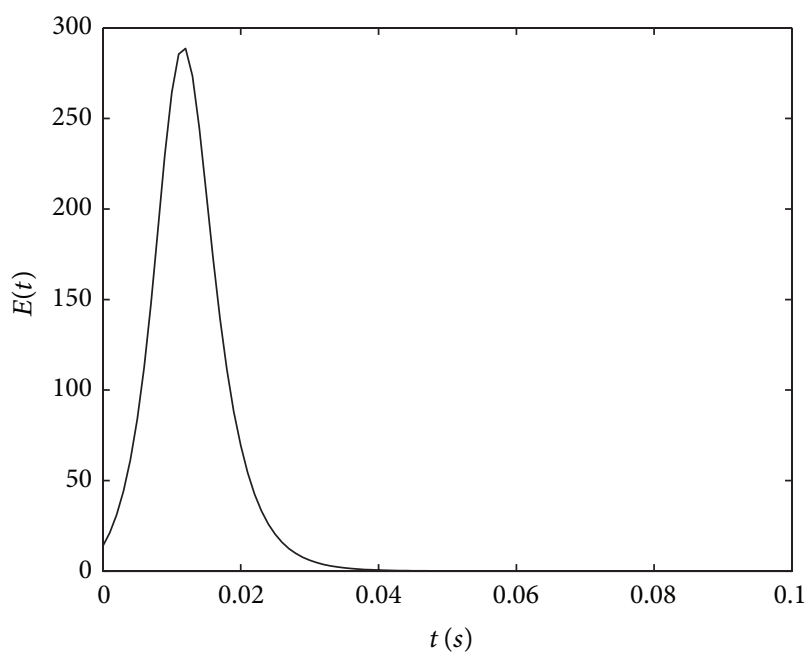

Figure 3: Time evolution of $E(t)$.

of fractional-order complex network systems by employing fractional-order stability theory. Based on this scheme, the new adaptive pinning controllers are designed to realize the synchronization in complex networks and the node selected algorithm is given. In the simulation, the fractional-order Liu chaotic system as nodes for constructing network model and the Adams-Bashforth-Moulton algorithm are applied to the fractional differential equations of Liu chaotic system in the networks. The suitable adaptive feedback control gain adding to the pinned nodes for achieving all the nodes synchronized with each other in the complex networks. 


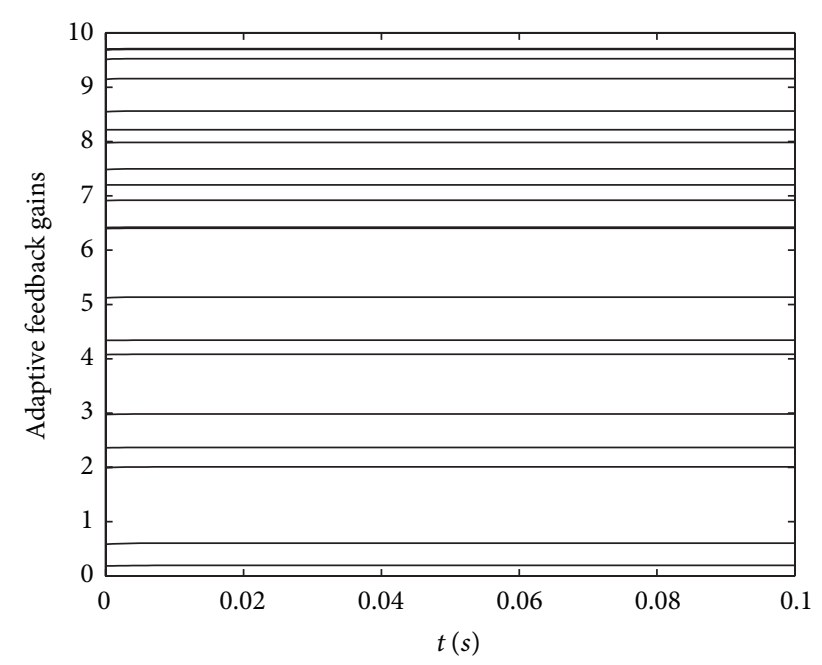

FIGURE 4: Adaptive feedback gains.

\section{Conflict of Interests}

The authors declare that there is no conflict of interests regarding the publication of this paper.

\section{Acknowledgments}

This paper is supported by the National Natural Science Foundation of China (nos. 51177117 and 51307130), the Creative Research Groups Fund of the National Natural Science Foundation of China (no. 51221005), and the Research Fund for the Doctoral Program of Higher Education of China (no. 20100201110023).

\section{References}

[1] I. Podlubny, Fractional Differential Equations, Academic Press, New York, NY, USA, 1999.

[2] R. Hilfer, Applications of Fractional Calculus in Physics, World Scientific, New Jersey, NJ, USA, 2001.

[3] P. L. Butzer and U. Westphal, An Introduction to Fractional Calculus, World Scientific, Singapore, 2000.

[4] R. C. Koeller, "Application of fractional calculus to the theory of viscoelasticity," Journal of Applied Mechanics, vol. 51, no. 2, pp. 299-307, 1984.

[5] H. H. Sun, A. A. Abdelwahab, and B. Onaral, "Linear approximation of transfer function with a pole of fractional order," IEEE Transactions on Automatic Control, vol. 29, no. 5, pp. 441-444, 1984.

[6] T. T. Hartley, C. F. Lorenzo, and H. K. Qammer, "Chaos in a fractional order Chua's system," IEEE Transactions on Circuits and Systems I: Fundamental Theory and Applications, vol. 42, no. 8, pp. 485-490, 1995.

[7] C. Li and G. Chen, "Chaos and hyperchaos in the fractionalorder Rössler equations,” Physica A, vol. 341, pp. 55-61, 2004.

[8] I. Grigorenko and E. Grigorenko, "Chaotic dynamics of the fractional Lorenz system," Physical Review Letters, vol. 91, no. 3, Article ID 034101, 4 pages, 2003.
[9] C. Li and G. Peng, "Chaos in Chen's system with a fractional order," Chaos, Solitons and Fractals, vol. 22, no. 2, pp. 443-450, 2004.

[10] X.-Y. Wang and M.-J. Wang, "Dynamic analysis of the fractional-order Liu system and its synchronization," Chaos, vol. 17, no. 3, Article ID 033106, 6 pages, 2007.

[11] X.-Y. Wang and J.-M. Song, "Synchronization of the fractional order hyperchaos Lorenz systems with activation feedback control," Communications in Nonlinear Science and Numerical Simulation, vol. 14, no. 8, pp. 3351-3357, 2009.

[12] X. Y. Wang and Y. J. He, "Projective synchronization of fractional order chaotic system based on linear separation," Physics Letters A, vol. 372, no. 4, pp. 435-441, 2008.

[13] X.-Y. Wang, Y.-J. He, and M.-J. Wang, "Chaos control of a fractional order modified coupled dynamos system," Nonlinear Analysis: Theory, Methods and Applications, vol. 71, no. 12, pp. 6126-6134, 2009.

[14] R. Albert and A. L. Baravasi, "Statistical mechanics of complex networks," Reviews of Modern Physics, vol. 74, pp. 47-97, 2002.

[15] L. M. Pecora, T. L. Carroll, and G. A. Johnson, "Fundamentals of synchronization in chaotic systems, concepts, and applications," Chaos, vol. 7, no. 4, pp. 520-543, 1997.

[16] G. Chen, J. Zhou, and S. Čelikovský, “On LaSalle’s invariance principle and its application to robust synchronization of general vector Liénard equations," IEEE Transactions on Automatic Control, vol. 50, no. 6, pp. 869-874, 2005.

[17] A. Barrat and M. Weigt, "On the properties of small world networks," European Physical Journal B, vol. 13, no. 3, pp. 547$560,2000$.

[18] M. E. J. Newman, "The structure and function of networks," Computer Physics Communications, vol. 147, no. 1-2, pp. 40-45, 2002.

[19] M. E. J. Newman, C. Moore, and D. J. Watts, "Mean-field solution of the small-world network model," Physical Review Letters, vol. 84, no. 14, pp. 3201-3204, 2000.

[20] R. Cohen and S. Havlin, "Scale-free networks are ultrasmall," Physical Review Letters, vol. 90, no. 5, Article ID 058701, 4 pages, 2003.

[21] A. Fronczak, P. Fronczak, and J. A. Holyst, "Mean-field theory for clustering coefficients in Barabási-Albert networks," Physical Review E, vol. 68, no. 4, Article ID 046126, 4 pages, 2003.

[22] M. Chen, "Chaos synchronization in complex networks," IEEE Transactions on Circuits and Systems I: Regular Papers, vol. 55, no. 5, pp. 1335-1346, 2008.

[23] X. Wu and H. Lu, "Cluster synchronization in the adaptive complex dynamical networks via a novel approach," Physics Letters A, vol. 375, no. 14, pp. 1559-1565, 2011.

[24] J. Zhou, J.-A. Lu, and J. H. Lu, "Pinning adaptive synchronization of a general complex dynamical network," Automatica, vol. 44, no. 4, pp. 996-1003, 2008.

[25] H. Nie, L. Xie, J. Gao, and M. Zhan, "Projective synchronization of two coupled excitable spiral waves," Chaos, vol. 21, no. 2, Article ID 023107, 7 pages, 2011.

[26] B. Liu, X. Liu, G. Chen, and H. Wang, "Robust impulsive synchronization of uncertain dynamical networks," IEEE Transactions on Circuits and Systems I: Regular Papers, vol. 52, no. 7, pp. 1431-1441, 2005.

[27] Y. Chai, L. P. Chen, R. C. Wu, and J. Sun, "Adaptive pinning synchronization in fractional-order complex dynamical networks," Physica A, vol. 391, pp. 5746-5758, 2012. 
[28] L. P. Chen, Y. Chai, R. C. Wu, J. Sun, and T. D. Ma, "Cluster synchronization in fractional-order complex dynamical networks," Physics Letters A, vol. 376, pp. 2381-2388, 2012.

[29] X. Wu, D. Lai, and H. Lu, "Generalized synchronization of the fractional-order chaos in weighted complex dynamical networks with nonidentical nodes," Nonlinear Dynamics, vol. 69, pp. 667-683, 2012.

[30] M. A. Mohammad, M. Joaquin, T. Mohammad, B. Hamidi, and S. T. Mohammad, "Robust outer synchronization between two complex networks with fractional order dynamics," Chaos, vol. 21, no. 3, Article ID 033121, 2011.

[31] D. Matignon, "Stability results of fractional differential equations with applications to control processing," IMACS, IEEESMC, Lille, France, 1996.

[32] J.-B. Hu, Y. Han, and L.-D. Zhao, "A novel stablility theorem for fractional systems and its applying in synchronizing fractional chaotic system based on back-stepping approach," Acta Physica Sinica, vol. 58, no. 4, pp. 2235-2239, 2009.

[33] C.-M. Qin, N.-M. Qi, and K. Zhu, "State space modeling and stability theory of variable fractional order system," Control and Decision, vol. 26, no. 11, pp. 1757-1760, 2011 (Chinese).

[34] X. F. Wang and G. Chen, "Synchronization in small-world dynamical networks," International Journal of Bifurcation and Chaos, vol. 11, no. 1, pp. 187-192, 2002.

[35] X. F. Wang and G. Chen, "Synchronization in scale-free dynamical networks: robustness and fragility," IEEE Transactions on Circuits and Systems I: Fundamental Theory and Applications, vol. 49, no. 1, pp. 54-62, 2002.

[36] W. Yu, G. Chen, and J. Lu, "On pinning synchronization of complex dynamical networks," Automatica, vol. 45, no. 2, pp. 429-435, 2009.

[37] S. Boyd, L. E. Ghaoui, E. Feron, and V. Balakrishnan, Linear Matrix Inequalities in System and Control Theory, SIAM, Philadelphia, Pa, USA, 1994.

[38] K. Diethelm and N. J. Ford, "Analysis of fractional differential equations," Journal of Mathematical Analysis and Applications, vol. 265, no. 2, pp. 229-248, 2002. 


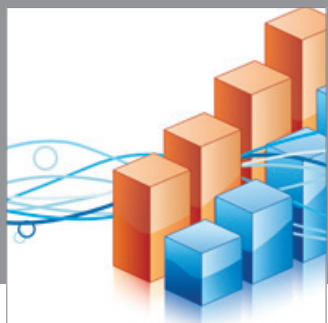

Advances in

Operations Research

mansans

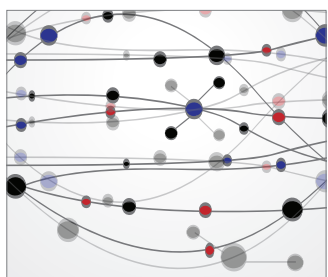

The Scientific World Journal
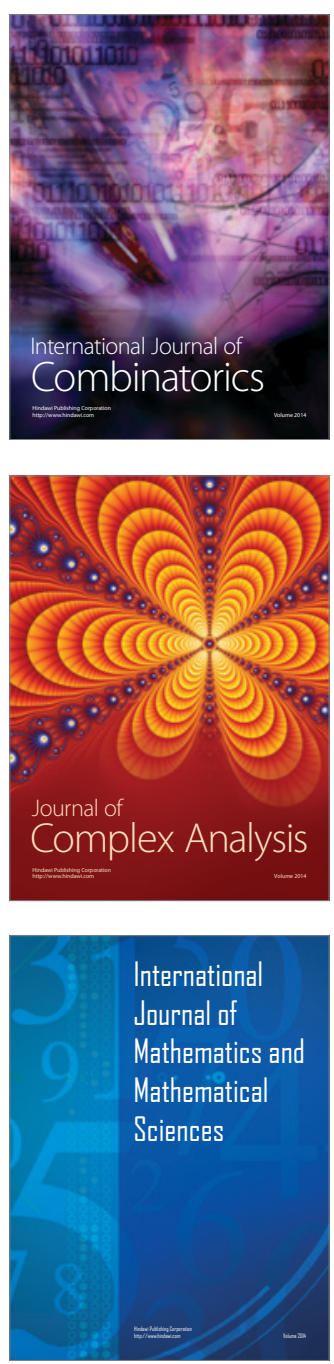
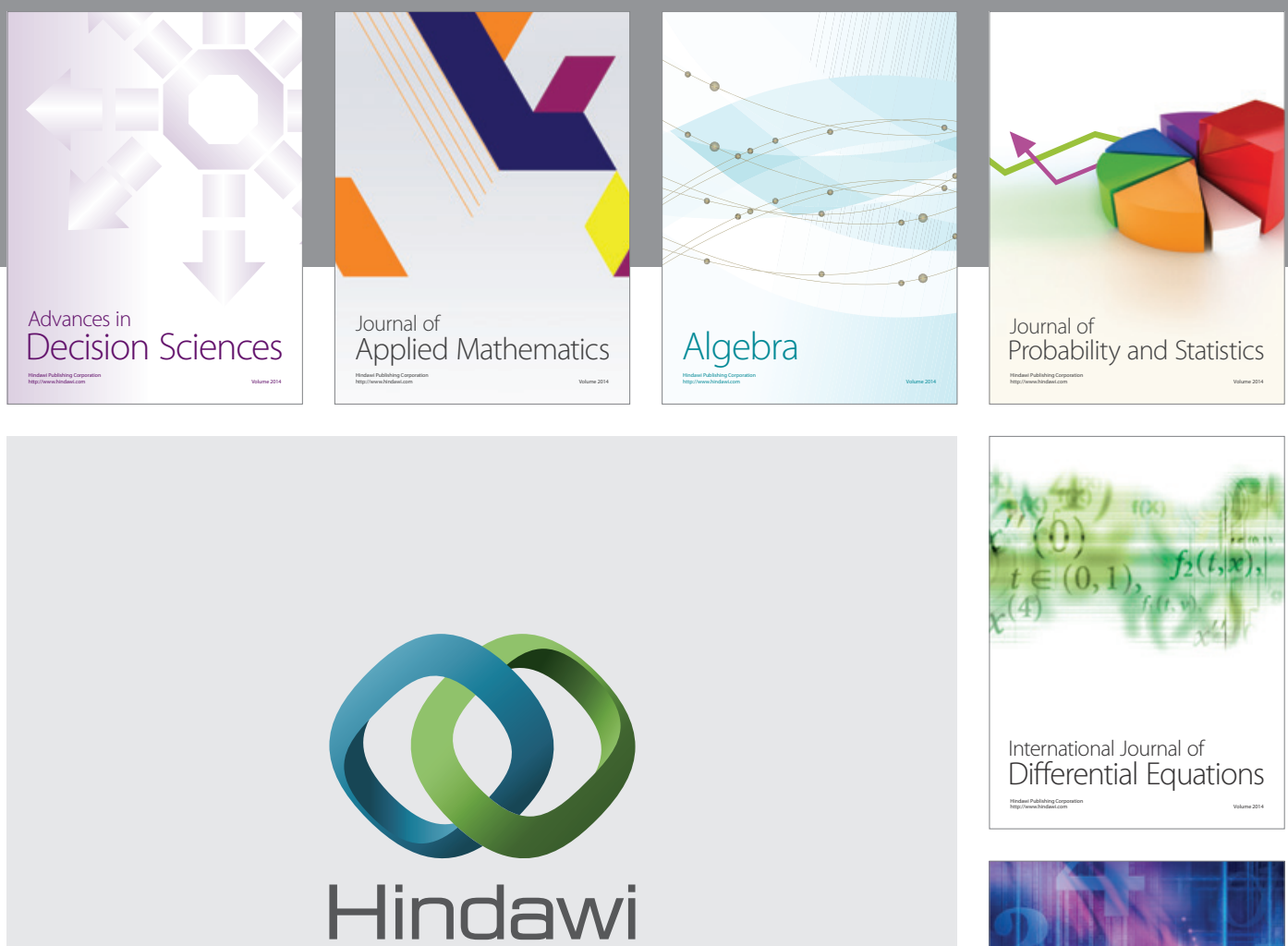

Submit your manuscripts at http://www.hindawi.com
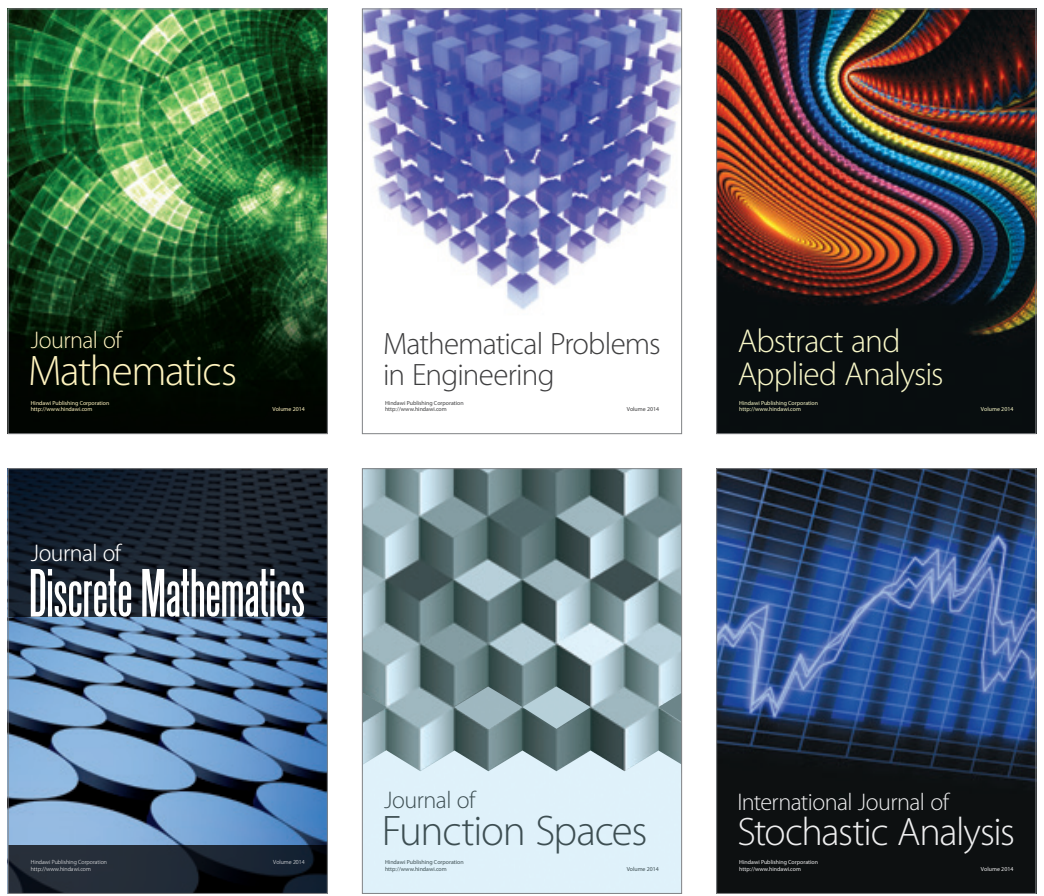

Journal of

Function Spaces

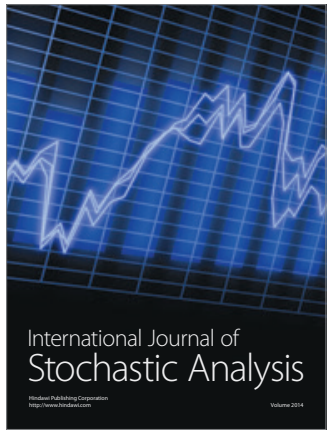

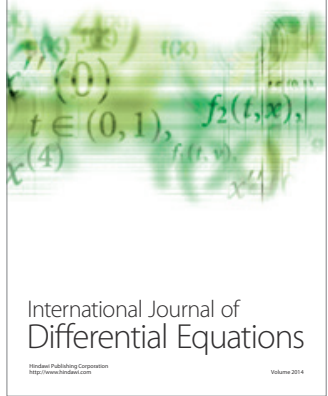
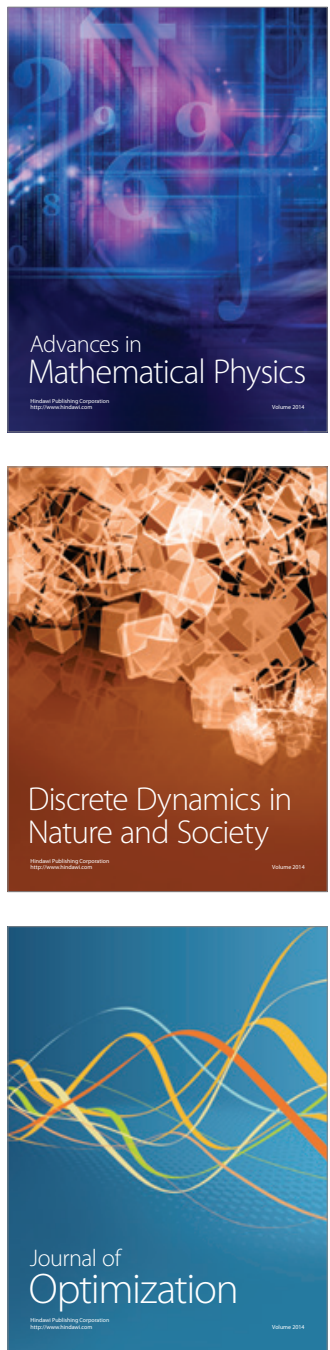